\title{
Uniform crashworthiness optimization of car body for high-speed trains
}

\author{
Mirosław Mrzygłód • Tomasz Kuczek
}

Received: 13 March 2012 / Revised: 8 July 2013 / Accepted: 9 July 2013 / Published online: 1 September 2013

(C) Springer-Verlag Berlin Heidelberg 2013

\begin{abstract}
The paper deals with a new uniform crashworthiness concept of car bodies optimization of high-speed trains. The design optimization was done from the point of view of structural protection of occupants' survival space. For the reason that it is impossible to find a highly probable scenario for the derailment, the authors decided to find the solution in the form of rigid frame structure (survival cells), which will provide safety space for the passengers.

In the optimization example a typical passenger car body was divided into cells of approximately equal dimensions. The optimization problem was to minimize the mass of the structure with stress constraints. The survival cell was subjected to a sequence of high value loads. The loads are acting in an asynchronous way in three load directions what gives the optimized structure uniform crashworthiness.

The optimization strategy consists of three stages. In the first step, the constant criterion surface algorithm (CCSA) of topology optimization is applied to find a preliminary solutions. For improving the manufacture properties of this solution, a new concept of design space constraints was proposed. The sizing optimization with evolutionary algorithms was used to define a thin-walled structure in the second step. For evolutionary optimization a standard procedure was employed. Finally, CCSA optimization algorithm was
\end{abstract}

This paper was presented in the 9th World Congress on Structural and Multidisciplinary Optimization, International Society for Structural and Multidisciplinary Optimization, Shizuoka, Japan, 2011

M. Mrzygłód · T. Kuczek $(\bowtie)$

Institute of Rail Vehicles, Cracow University of Technology, al. Jana Pawła II 37, 31-864, Kraków, Poland

e-mail:kuczek@m8.mech.pk.edu.pl

M. Mrzygłód

e-mail: mrzyglod@mech.pk.edu.pl applied again to remove excessive material from a car body structure. As the optimization result a new design proposition of a car body with multiple survival cells of high uniform stiffness was obtained. By maintaining passengers' survival space, the passive safety of a high-speed car body was significantly increased.

Keywords Uniform crashworthiness - Passive safety of high-speed trains · Topology optimization · Design space constraints

\section{Introduction}

High-speed train cars produced at present usually fulfill all structural requirements. However, in the real accidents of a high-speed operation, the vehicle design does not ensure sufficient safety for passengers. It is especially visible in case of derailments and side crashes with railway infrastructure (Iselius et al. 2006). According to empirical data, the probability of derailment increases with a train crossing at a speed of over $70 \mathrm{~km} / \mathrm{h}$ (Brabie and Andersson 2008).

Crashworthiness can be defined as the ability of a structure to protect occupants from injuries during an impact. Current crashworthiness requirements for car body design can ensure passenger safety only for low speed vehicle operations. For example, Euronorms assume that during collision a train will have the speed $\mathrm{Vc}=36 \mathrm{~km} / \mathrm{h}$ (CEN 2008). Moreover, they recommend equipping the vehicle ends with energy absorbers that will be designed for a crash with an identical car at the speed of $0.5 \mathrm{Vc}$ (CEN 2008). Safety has to ensure also high stiffness of the structure, that should transfer a static load of Fs $=2 \mathrm{MN}$ working along the axis of the car underframe (CEN 2002). The crashworthiness requirements consider the highest 
passive safety only for passenger compartments located in the leading vehicle of the trains. The detailed analysis of crashworthiness requirements and the verification procedure for high-speed vehicles was presented in the work of Kirkpatrick et al. (2001). Xue et al. (2007) have summarised the present structural designs of passenger cars and their influence on crash resistance. Martinez et al. (2004) propose the conception of a car with passenger safety space that is designed in case of crash incidents of derailments. He takes into account the strengthening of the front walls and roof by introducing energy absorbing zones.

The aim of this work is to find a new methodology of crashworthiness design of passenger cars by applying structural optimization. The new design should fulfill all requirements of current standards and be characterized also by high resistance to side impact in case of derailment. It is impossible to find a highly probable scenario for the derailment (CEN 2008). Thus, the authors decided to find the solution in the form of rigid frame structure, which will provide safety space for the passengers. The use of modern methods and the tools for structural optimization should make it possible to improve passive safety without increasing vehicle mass. To ensure high side impact endurance, the car body structure will be divided into several sub-systems. Each of the structural sub-systems will define passengers' survival cell (PSC) of an equal stiffness resistance. The front part of the car body will be excluded from structural optimization as a place of energy absorbers location (CEN 2008).

The optimization of PSC will be carried out in three stages. In the first stage preliminary topology optimization will be conducted by using a design space constraints. In the second stage, the parametric model of the structure will be used. The parametric model will be built taking into consideration the results of the first stage optimization. The first stage finite element model of solid type elements will be replaced by an equivalent FE model of shell type elements. The shell thickness of the FE model will become parameterized. This model is to be optimized by using genetic algorithms (GA). The solution obtained from the GA optimization will be a starting point for the third stage of optimization. In that stage, the unnecessary material from the thin-walled FE model will be removed through topology optimization.

\section{Constant criterion surface algorithm}

For topology optimization of stages I and III, the constant criterion surface algorithm (CCSA) was selected (Mrzygłód 2009: 2012). The optimization problem of the CCSA algorithm can be formulated as follows:

$\min _{\eta} f\left(\eta_{i}\right)$ the constraints are:

$g_{j}\left(x_{i}\right) \leq \overline{g_{j}} ; \quad j=[1,2, \ldots, K]$

where: $x_{i}$ is a vector of finite elements $(i=[1,2, \ldots, N])$; $\eta_{i}$ is a vector of design variables defined as $\eta_{i}=E_{\min } / E_{0}$, $E_{\min }$ and $E_{0}$ respectively, minimum and real material Young's modules; $g_{j}\left(x_{i}\right)$ are the constraints (e.g. the equivalent stress, compliance or fatigue); $\overline{g_{j}}$ are the upper bounds of constraints; $f\left(\eta_{i}\right)$ is the objective function (the volume of structure). The design variables $\eta_{i}$ (pseudo-density) represent stiffness of each finite element of the structures that vary between $E_{\min }$ and $E_{0}$. The lower boundary of stiffness $E_{\min }$ is introduced to prevent singularity of the equilibrium problem.

The homogenization method (Bendsøe and Kikuchi N 1988), SIMP (solid isotropic material with penalization) (Zhou and Rozvany 1991) and ESO/BESO (evolutionary structural optimization/ bidirectional evolutionary structural optimization) (Querin et al. 1998; Xie and Steven 1993) are popular methods of topology optimization (Bendsøe and Sigmund 2003). The CCSA algorithm is a "hard-kill" type method, in which the volume value of optimized structure is not assumed a priori. The solution in this method is generated by iterative elimination of low value elements of a criterion function $g$ (see Fig. 1). In result of such an action the surface of constant constraint criterion is obtained in the optimized structure. The idea of shaping structures in the form of the surface of constant stresses was first proposed by Mattheck and Burkhardt (1990). However, the condition of constant energy density at the free surface of the optimized structure was first derived by Wasiutynski (1960).

The procedure of iterative elimination of low stressed elements was first introduced by Xie and Steven (1993) in ESO method. In the ESO algorithm, the optimization process is controlled by rejection rate parameter that constantly increases the level of stress limit for element elimination. In comparison to this, the CSSA removal procedure is controlled by a constant parameter of volume percentage reduction $\Delta F$, which gives a possibility to control the optimization "speed" as well as to stabilize the optimization process near the quasi optimum. To find the $\Delta F$ value, a constraint criterion limit $g_{\min }$ at every volume decreasing iteration is dynamically calculated. The FE elements having values of constraint criterion parameters $g$ below the $g_{\text {MIN }}$ limit are eliminated from the structure.

The topology optimization procedure can give premature results when it is stuck in the point of high values of the state parameter. In the CCSA algorithm when criterion function is over the limit, a layer of finite elements is added to the entire boundary of the structure (see Fig. 2). For the volume increasing iteration there is no removal operation. 


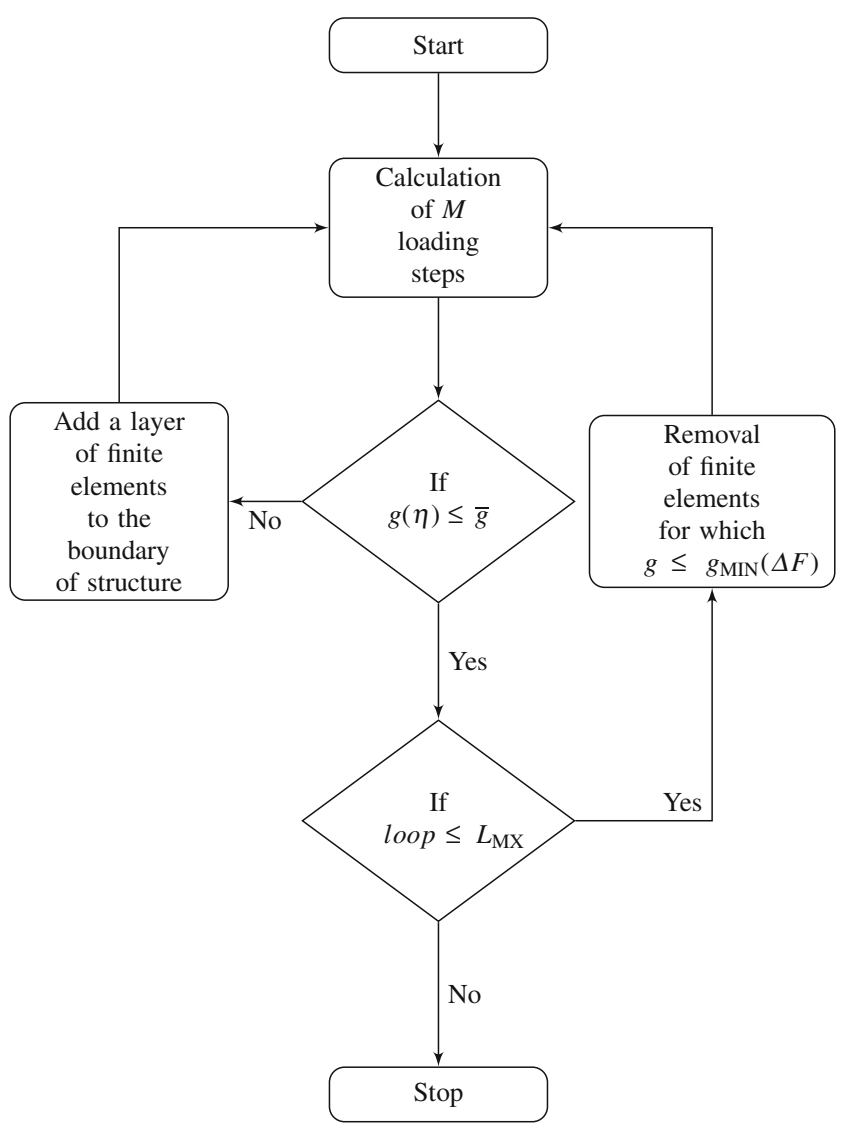

Fig. 1 The constant criterion surface algorithm

The procedure of increasing the volume of the structure is continued until the criterion parameter $g$ returns to admissible values. By increasing and decreasing the structure volume, the algorithm obtains better solutions. This scheme is analogous to the simulated annealing (SA) (Kirkpatrick et al. 1983).

In the CCSA method, the forces that act in an asynchronous way on the structure are taken into account by a 'compare and save maximum' procedure of summation of
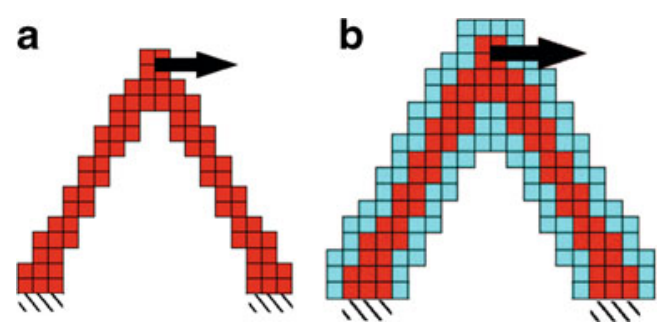

Fig. 2 The layer expansion algorithm: a structure before (a) and after operation of adding a layer of finite elements to the structure boundary (b) constrain criterion values (Mrzygłód 2010). The procedure assumes that during each iteration for every finite element only the maximum values of the constraint criterion of all load cases will be written to the equivalent design space. The constraint values of final equivalent design space are used by the constant criterion surface algorithm of topology optimization. For multi-constrained topology optimization problems normalized constraints are introduced (Mrzygłód 2012).

To test the convergence of the algorithm, the benchmark problem of optimizing truss topology with application of compliance constraint was selected (Rozvany 1998) (see Fig. 3a-b). In the numerical example, the design domain is discretized with $160 \times 404$-node plane stress elements. The Young's modulus $E=1$, Poisson's ratio $v=0.3$, load value $F=1$ and compliance constraint $\bar{g}=0.21$ are assumed.

The solution obtained by CCSA algorithm is consistent with the data published in literature for the compliance constraints (Bendsøe and Sigmund 2003; Huang and Xie 2010) (see Fig. 3c ). In Fig. 3d the history of searching for a solution is presented. The simulated annealing plot shows convergence of objective function (volume). Moreover, in the plot the values of constraints (compliance) and boundary for feasible solutions $(\bar{g})$ are presented.

In Fig. 3d we can see that only about $1 / 10$ of the volume that has been added is removed. The rest of the added volume (9/10) is used by algorithm as new search regions. In the CCSA algorithm we do not use sensitivity analysis and local rules to modify the structure like in SIMP, BESO or Cellular Automata (CA) methods (Tovar et al. 2006). However, the results of the test confirm efficiency of the layer expansion procedure in overcoming the local minima problem.

For the realization of the example as well as the topology optimization procedure the ANSYS APDL script language was used (ANSYS Inc 2010).

\section{Topology optimization with design space constraints}

The topology optimization of large thin-walled structures leads to solutions that can make problems in practical realization. When the design space of topological optimization is discretized by the finite elements of small dimensions, the optimization algorithm usually shapes the final solution in the form of thin rod structures with low usefulness for the production process (see Thomas et al. (2002)). For that reason a new design space preprocessing procedure was proposed.

The procedure consists of the discretization of the design space by solid type finite elements of a hexahedron shape and equal dimensions. Moreover, the equivalent stiffness is 
Fig. 3 The Rozvany benchmark problem (Rozvany 1998): problem description (a), analytical solution $(\mathbf{b})$, best solution $\mathrm{Vol}=53,21 \%$ (c), history of searching for a solution $\left(L_{\mathrm{MX}}=200\right)(\mathbf{d})$ a

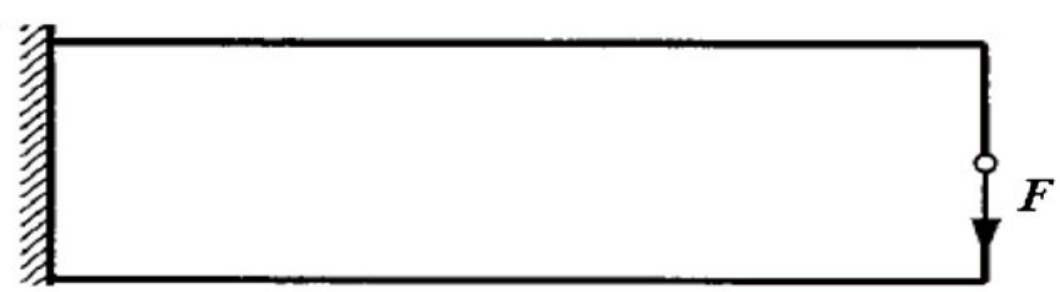

b

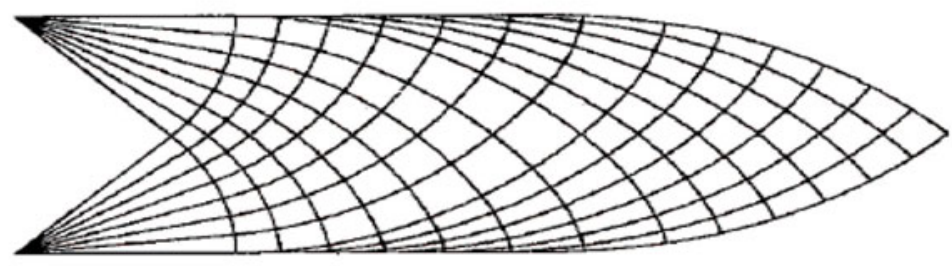

C

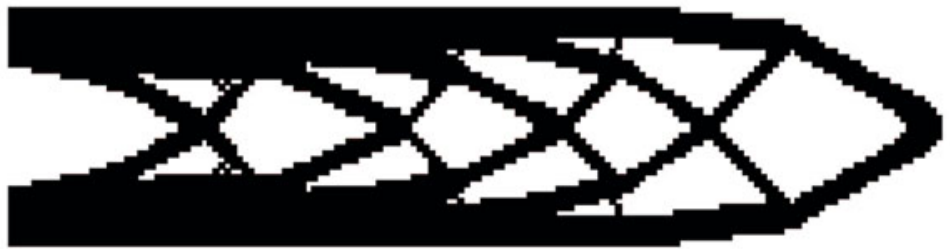

d

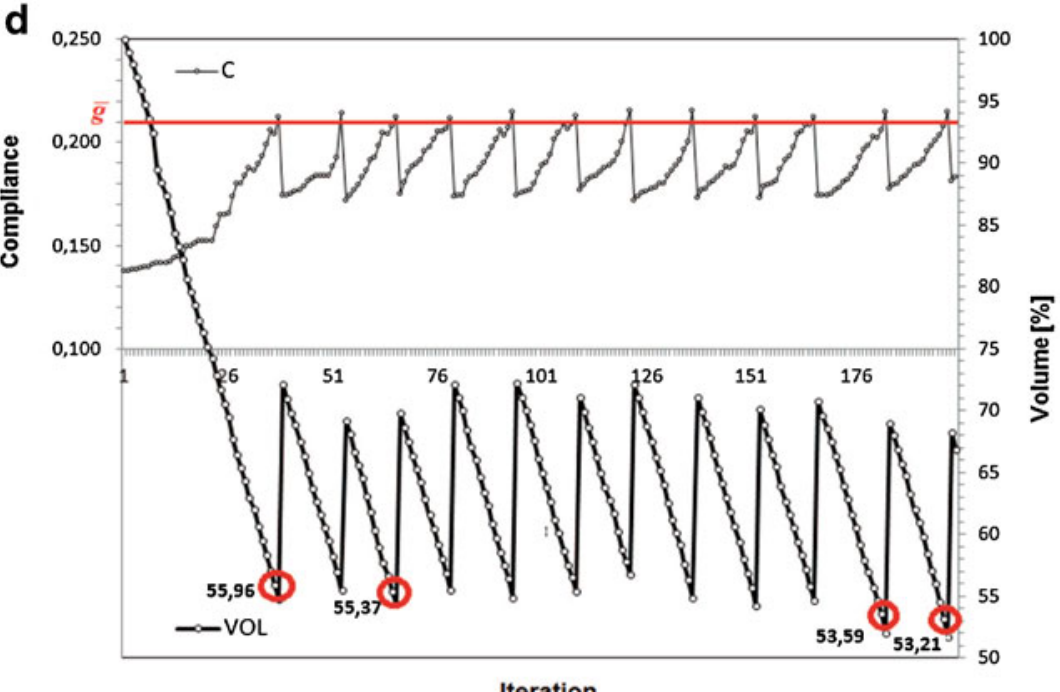

Iteration assigned to the finite elements. The equivalent stiffness is calculated from the thin-walled structure (e.g. a cube made of thin sheet) that has the same dimensions as the dimensions of finite elements of the design space. This allows to impose the topological constraints on the final solution of topology optimization.

To illustrate the concept of design space constraints an example of a simple structure bracket subjected to a two asynchronous loads was used (see Fig. 4a). Figure 4b presents the result of topological optimization with design space, that is discretized by finite elements of small dimensions. As can be clearly seen, the optimum structure has got the layout not suitable for use in case of a structure made of thin-walled profiles.
In Fig. 4c-i the procedure of pre-processing of the design space is presented.

In the first step, the finite element model of a thinwalled cube loaded by a unit force is calculated (Fig. 4c-d) for obtaining the value of linear deformation (Fig. 4e). The dimensions of the cube are selected on the basis of cross-sectional dimensions of a standard profile to be used for the design. In the next step, a solid finite element model with the same external dimensions and load conditions is prepared (Fig. 4f-g). The stiffness of this model $E_{0}$ is to be tuned to reach the value of displacement equal to the first model. The results of the displacement tuning are presented in Fig. 4h-i (compare Fig. 4e). In the last step, the design space is divided into finite 
Fig. 4 The concept of design space constraints procedure: an example of bracket problem (a), the result of topological optimization without using design space constraints (b); a thin-walled cube (c) and its FE model $(\mathbf{d})$, the value of linear deformation for thin-walled cube (e); a solid cube (f) and its FE model (g), the value of linear deformation for solid cube (h) and results of stiffness tuning (i); the result of topology optimization with using design space constraints (j) a

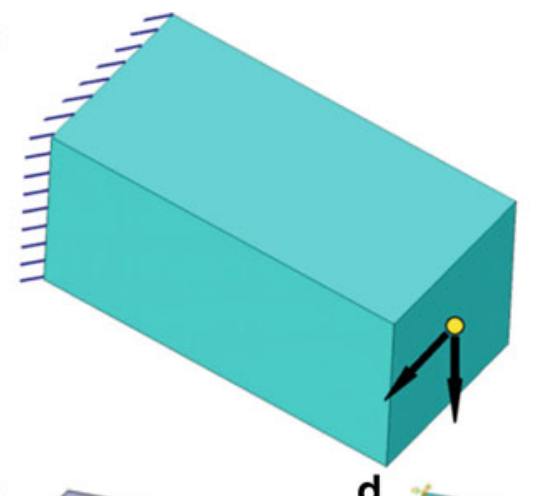

C
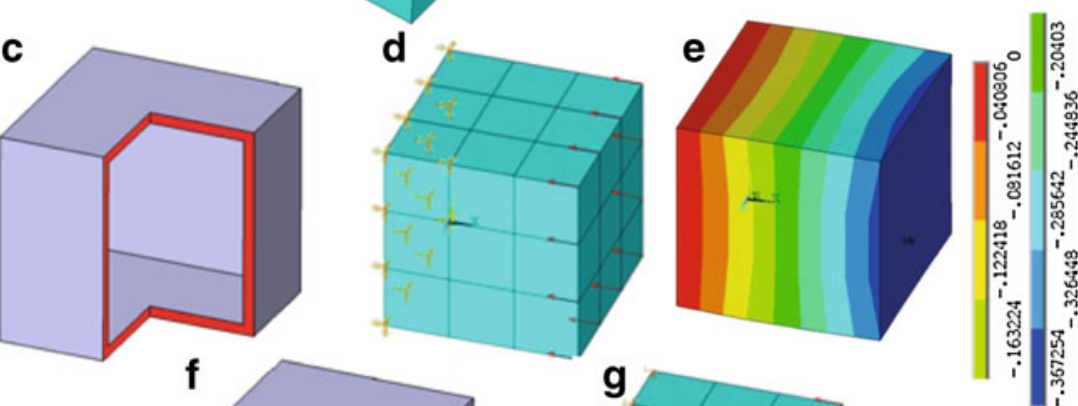

b

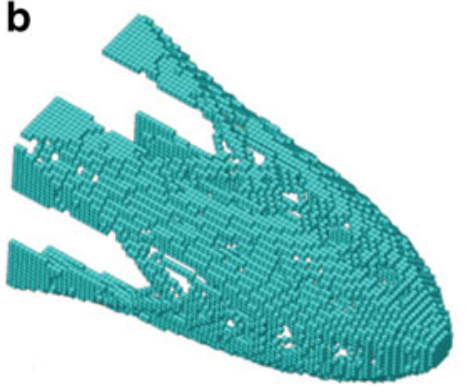

h
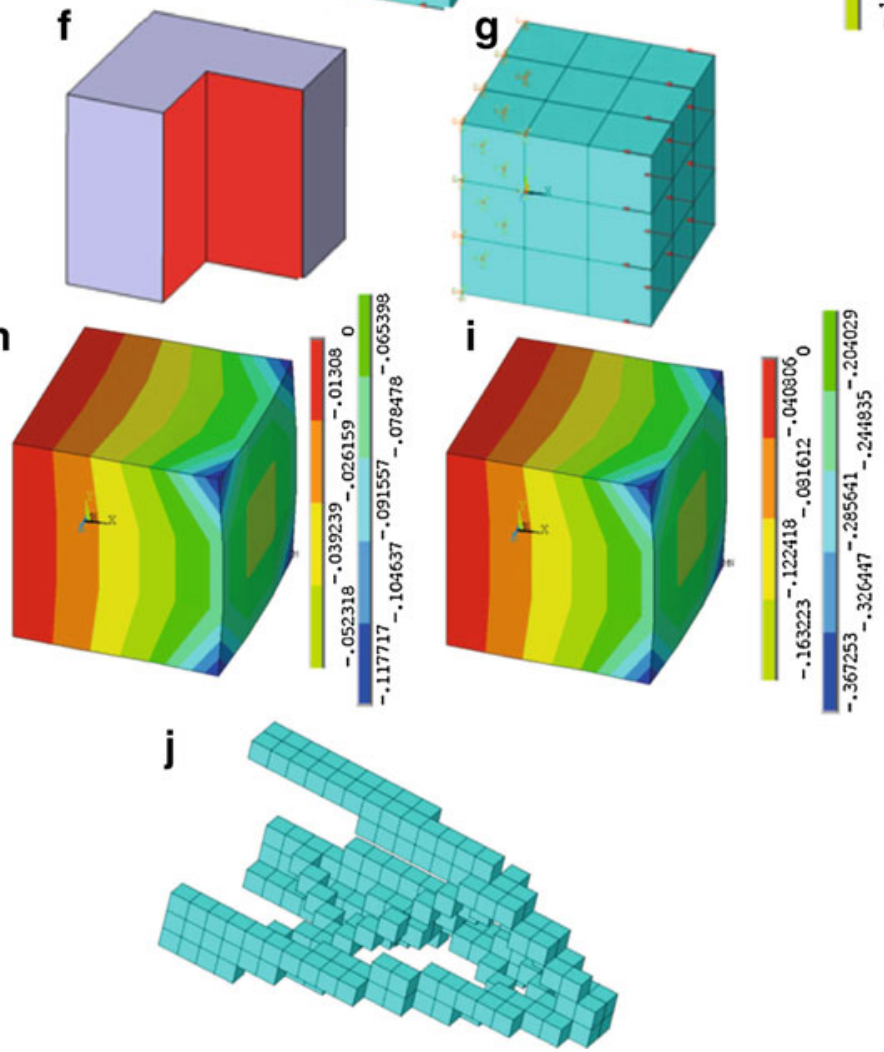

elements of the external dimensions of the cross-section of accepted standard profile. Moreover, the equivalent stiffness is assigned to all finite elements of the design space.

The solution of the topology optimization example with the procedure of design space pre-processing is shown in Fig. 4 .

\section{Example of crashworthiness optimization}

As an example of optimization of a typical passenger car body of a high-speed train was selected. The wagon was divided into cells of approximately equal dimensions (length $=$ breadth $=\sim 3000 \mathrm{~mm}$ ). For the simplification of a numerical model, only $1 / 4$ of the cell will be considered 
with symmetry boundary conditions. The FE model shown in Fig. 5a was prepared by using ANSYS software (ANSYS Inc 2010). In Fig. 5b-e boundary conditions and loads of analysis and optimization were presented. These conditions will be identical for all stages of optimization. Apart from symmetry boundary conditions, a vertical restraint was also applied (Fig. 5b). The force of $F_{s}=2 \mathrm{MN}$ was accepted as the value of load steps (CEN 2008: 2002). The loads are acting in an asynchronous way in three load directions what gives the optimized structure uniform crashworthiness (see Fig. 5c-e).

As a design space for topology optimization the volume of a car body was assumed. The design space was discretized using solid finite elements of approximately 50x50x50 mm dimensions (see Fig. 5). The topology optimization problem was to minimize the mass of the structure with stress constraints. The elastic limit of material
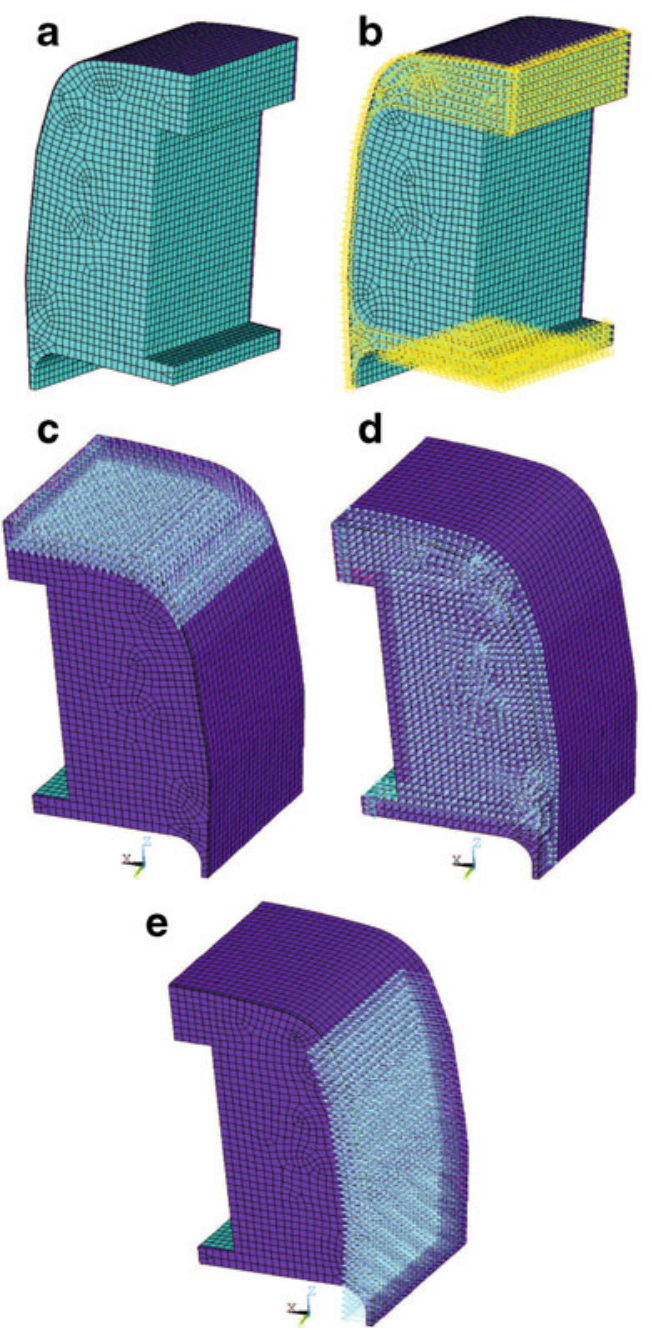

Fig. 5 The design space for topology optimization (a), boundary conditions of the FE model (b) and three load directions of stage I: Z (c), $\mathrm{Y}(\mathbf{d}), \mathrm{X}(\mathbf{e})$
(210 MPa of von Mises stress) was considered as a state parameter. The design space constraints were assumed for topology optimization and the thin-walled cubic FE model $(50 \times 50 \times 50 \times 2 \mathrm{~mm})$ was used to determine the equivalent stiffness of solid finite elements. The optimization 'speed' of CCSA algorithm was set on the level of $1 \%$ volume percentage reduction $\Delta F$. In Fig. 6 the result of the first stage of optimization is presented. The solution approaches a limit of constraint, what can be treated as a first quasi optimum. Because the finite element model is computationally expensive (high dimensional and multi-load step) the first quasi optimum solution was accepted for the next stage of optimization.

The external skin of a car body structure was excluded from optimization. The skin is considered as a multi-layer composite structure that is characterized by high resistance to tearing and penetration. The skin can be a subject of separate analysis and optimization. The numerical model of the skin in Fig. 5a is marked with a darker color. In the next stages of optimization the skin model will be omitted in the visualization.

For the second stage of optimization the result structure from the previous stage was transformed to a new FE model. The new model has a similar shape as in stage I and is characterized by a thin-walled structure.It is built with 4-node shell FE elements (see Fig. 7a). The elements of the FE model are divided into 20 groups of 20 thickness parameters. The shell FE model presented in Fig. 7 may look like a solid model due to the closure of all the shapes of the structure. Determination of parametric thickness for each zone of the shell model is visualized by different color of elements in Fig. $7 \mathrm{a}$ and c.

The parametric FE model was subjected to GA optimization by Evolutionary Optimization System (EOS) software (Osyczka et al. 2004). The optimization problem was to minimize the mass of the structure with stress constraints. For optimization, twenty design variables (thickness parameters) were accepted.

The GA optimization was conducted for 16 different "seed" numbers. Moreover, the following steering parameters of EOS were accepted (Osyczka et al. 2004): population size $J=200$, number of generation $N=125$, probability of crossover $p_{c}=0.7$, probability of mutation $p_{m}=0.4$. The EOS software was connected with the FE program ANSYS for performing batch processing values calculation of objective function and stress constraints (ANSYS Inc 2010). The best solution obtained from stage II is presented in Fig. 7b. The contour map of von Mises stresses for optimum structure of stage II are shown in Fig. 7c.

In the last stage, the FE model of a thin-walled structure obtained from EOS is subjected to topology optimization. For this purpose the CCSA algorithm will be used again. The topology optimization problem is to minimize the mass 
Fig. 6 History of searching for a solution (a) and final result of the first stage of optimization $\mathrm{Vol}=16,15 \%(\mathbf{b})$
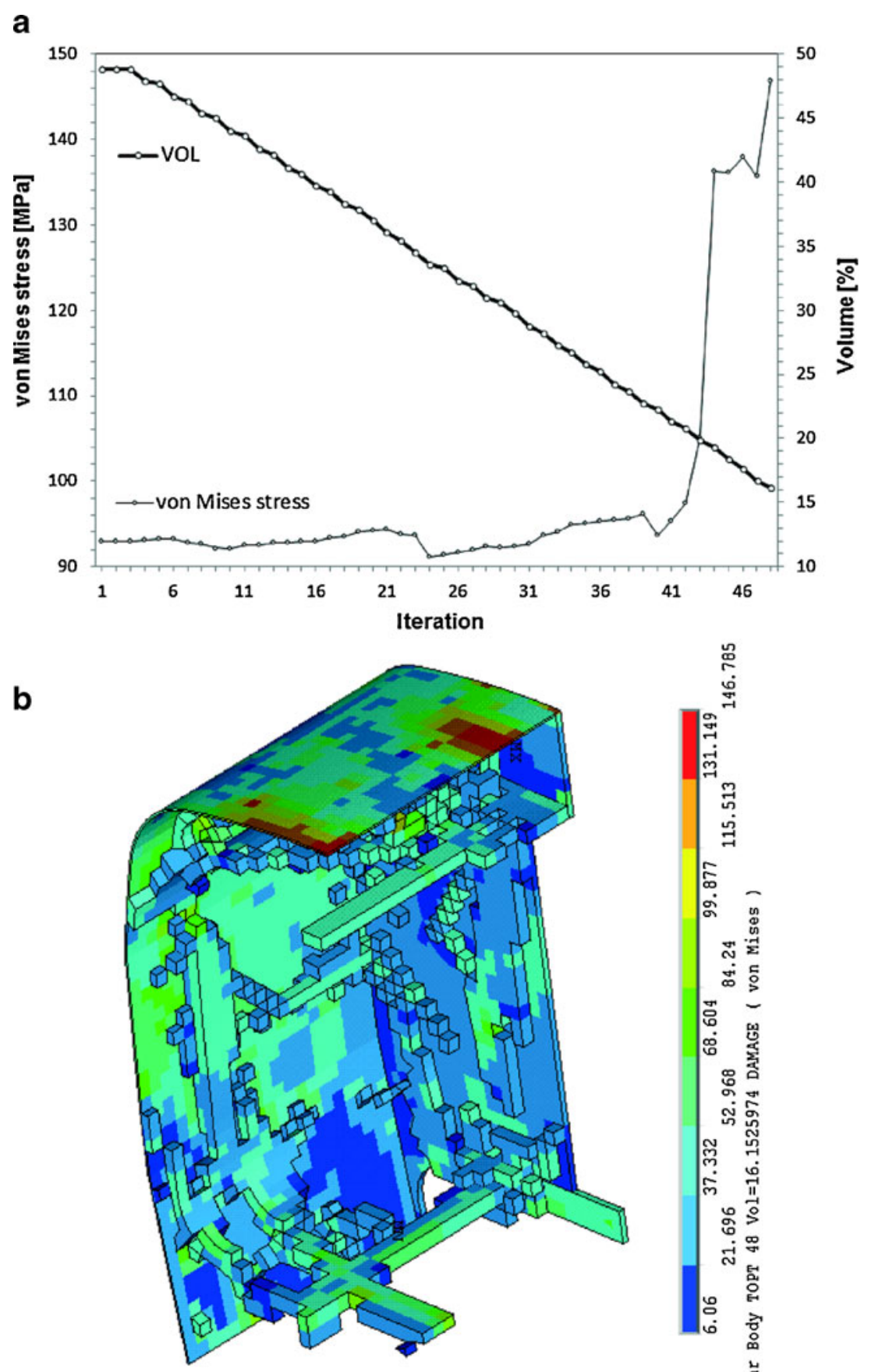

of the structure with stress constraints. The design space of optimization is limited by the FE model of stage II with twenty 'frozen' thickness parameters of the shell structure.

The third phase was based on the same FE model, but the number of variables is equal to the number of elements (approximately 30,000 of stiffness parameters of finite elements). The final results of topology optimization are presented in Fig. 8a-b. The objective function obtained in stage II was decreased by $13 \%$. The topology optimization applied in the last stage of optimization improved the result by 'removing' the excess material which clearly could not be removed by the sizing method. In Fig. 8a a history of searching for a solution is presented. For reasons similar to stage I, the first quasi optimum solution was accepted as the final solution of optimization. Figure $8 \mathrm{~b}$ shows the final FE model in which the 'unnecessary' walls were removed what resulting in changing the thin-walled structures into the plate type structures (see Fig. 8d). The result of the final stage of optimization in form of a CAD model is presented in Fig. 8c and d. 
Fig. 7 The FE shell model of thin-walled structure (the thickness parameters are visualised by colors and numbers) (a) and the best solution obtained of GA optimization from stage II (b) and its stress map of FE model (c)

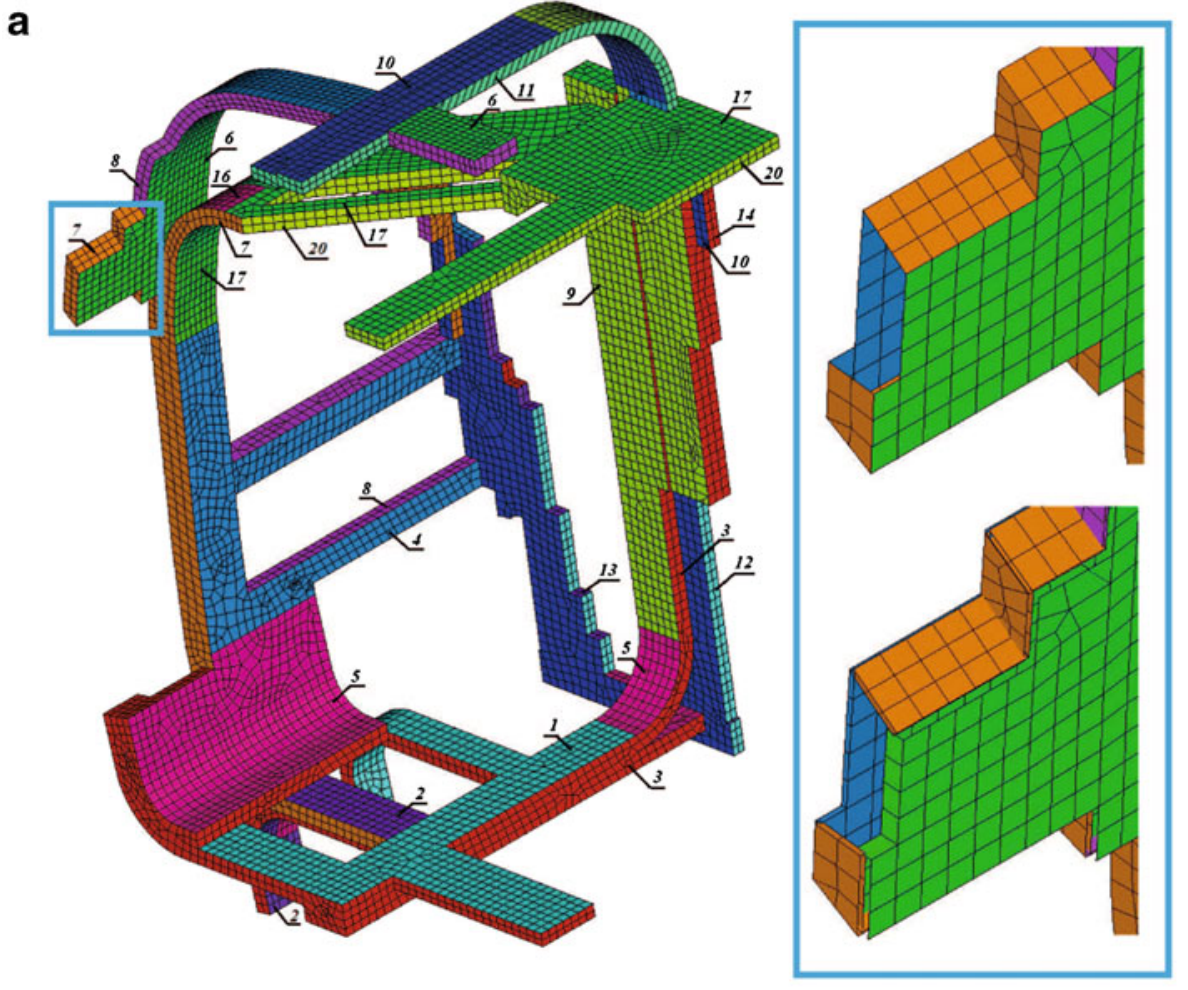

b

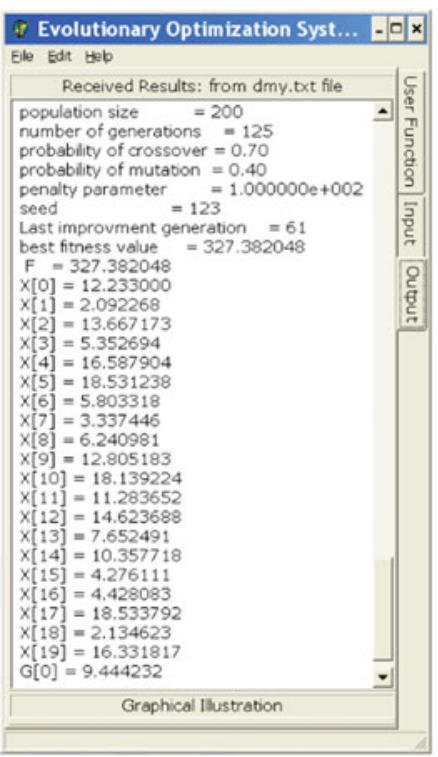

c

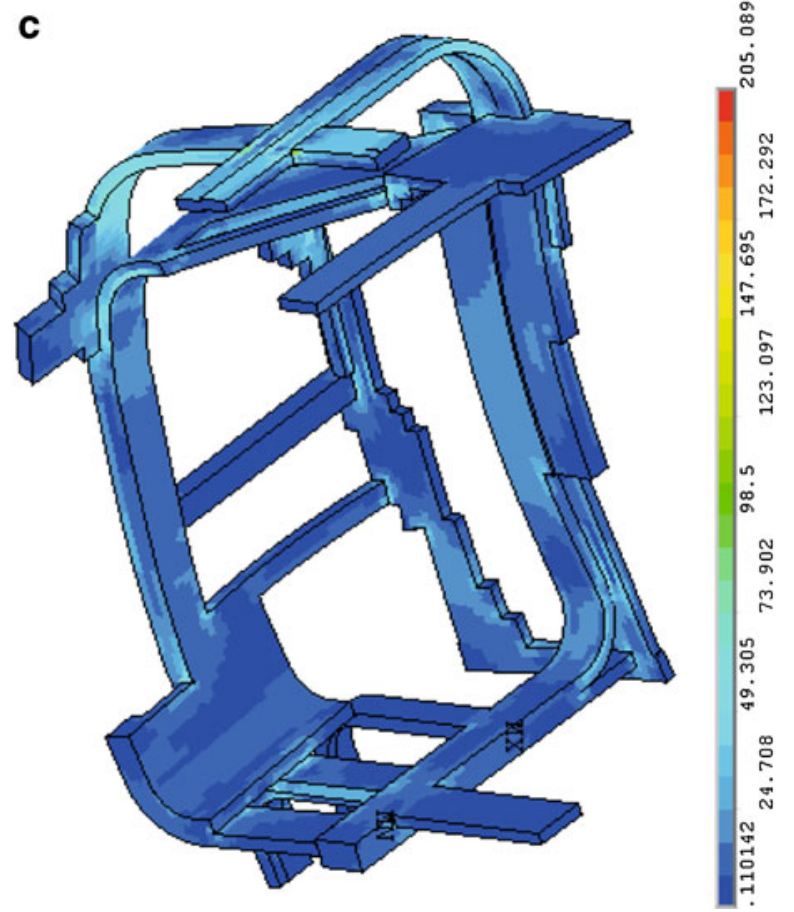

\section{Conclusions}

In the paper, a new concept of uniform crashworthiness optimization of a high-speed train car body using multiple survival cells is presented. In this complex optimization framework, the constant criterion surface algorithm of topology optimization with design space constraints as well as GA optimization based on sizing method were included. As shown in the example, the multi-stage methodology turned out to be an effective tool for crashworthiness optimization of rail vehicles. A new design proposition of a car body with multiple survival cells of uniform stiffness in 
Fig. 8 History of searching for a solution (a) and final result of topology optimization of stage III $\mathrm{Vol}=86,96 \%(\mathbf{b})$, the CAD model of passengers' survival cell $(\mathbf{d}, \mathbf{e})$

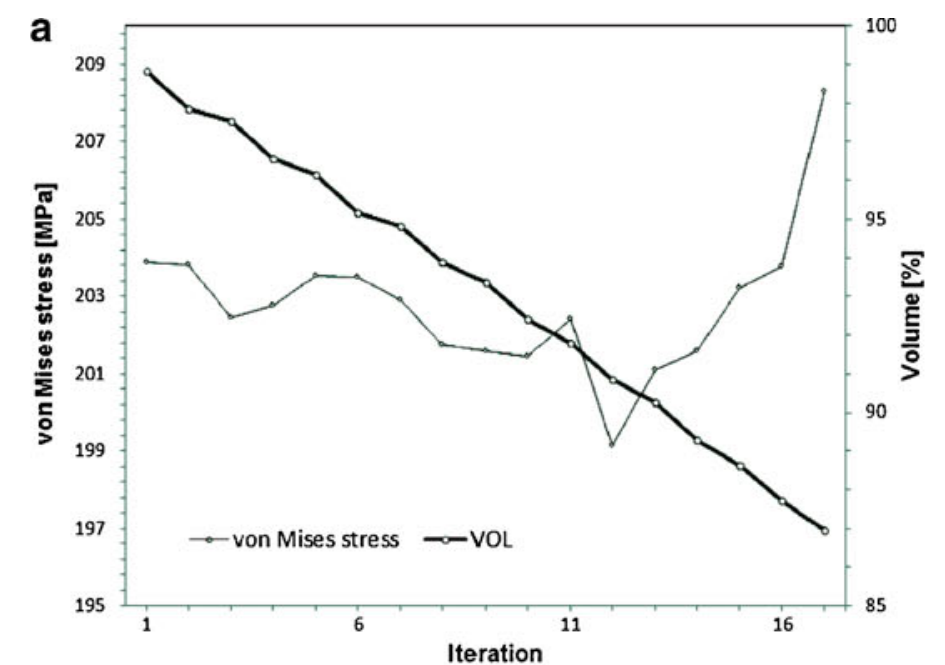

b

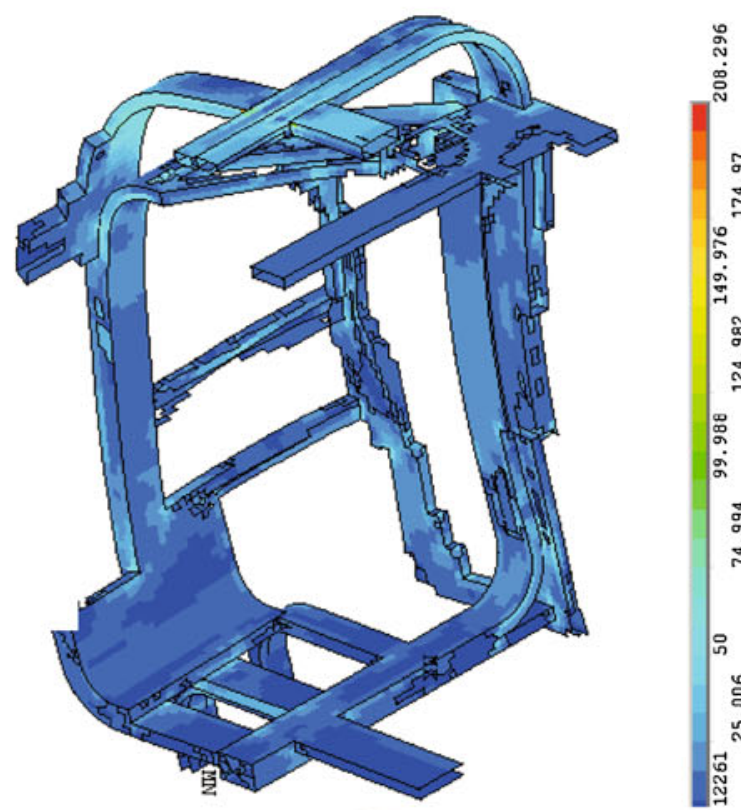

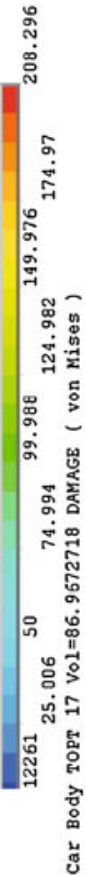

C

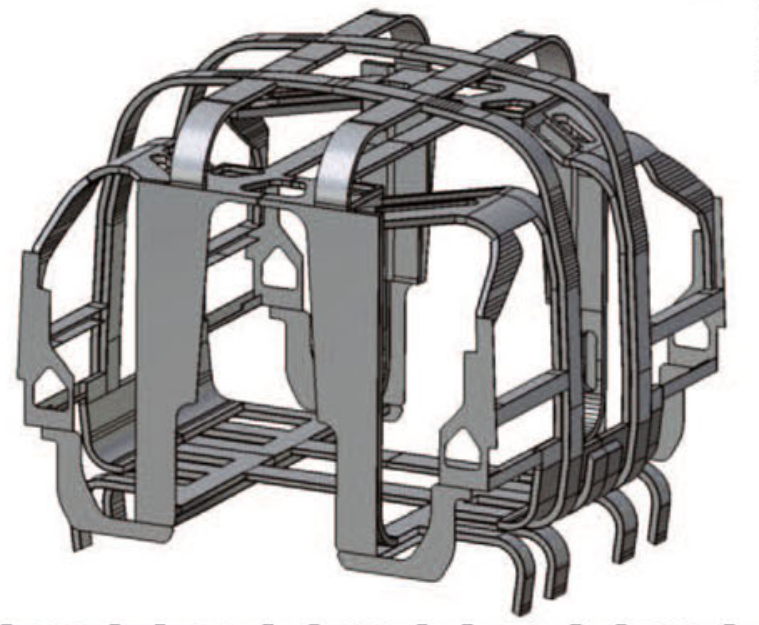

d

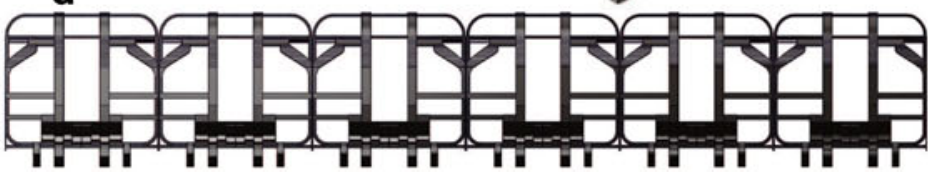


three directions was obtained from optimization. By maintaining passengers survival space, the passive safety of a high-speed car body was significantly increased. According to the safety requirements, the car structure can be tested by the calculation (CEN 2008: 2002). The verification of optimization results can be found in the fact that the structure under the load of $2 \mathrm{MN}$ remains within the elastic deformation stress level. The authors plan, as the next step of their research, to prepare the dynamic explicit tests of the passenger compartments with and without safety cages. However, the main effort will be given to the simulation of passengers' injuries.

The methodology presented in the paper can be easily extended to other types of railway vehicles.

\section{References}

ANSYS Inc (2010) Documentation for ANSYS, Release 13

Bendsøe MP, Kikuchi N (1988) Generating optimal topologies in structural design using a homogenization method. Comput Methods Appl Mech Eng 71:197-224

Bendsøe M, Sigmund O (2003) Topology optimization. Theory, methods and applications. Springer, New York

Brabie D, Andersson E (2008) An overview of some high-speed train derailments: means of minimizing consequences based on empirical observations. Proc IMechE, J Rail Rapid Transit 222:441463

CEN (2002) Railway applications-structural requirements of railway vehicle bodies. EN 12663

CEN (2008) Railway applications-crashworthiness requirements for railway vehicle bodies. EN 15227

Huang X, Xie YM (2010) A further review of ESO type methods for topology optimization. Struct Optim 41:671-683

Iselius L, Nilsson P, Riddez L (2006) Kamedo report no. 79: train accident in Germany 1998. Prehosp Disast Med 21(2):119-120

Kirkpatrick SW, Schroder M, Simons JW (2001) Evaluation of passenger vehicle crashworthiness. Int J Crashworthiness 6:95106

Kirkpatrick S, Gelatt CD, Vecchi MP (1983) Optimization by simulated annealing. Science 220:671-680
Martinez E, Tyrell D, Perlman B (2004) Development of crash energy management designs for existing passenger rail vehicles In: Proceedings ASME RTD, ASME international mechanical engineering congress

Mattheck C, Burkhardt S (1990) A new method of structural shape optimisation based on biological growth. Int J Fatigue 12(3):185190

Mrzygłód M (2009) Using layer expansion algorithm in topology optimization with stress constraints In: Proceedings CMM2009computing methods mechanics. The University Zielona Gora Press, pp 319-320

Mrzygłód M (2010) Two-stage optimization method with fatigue constraints for thin-walled structures. J Theor Appl Mech 48:567578

Mrzygłód M (2012) Multi-constrained topology optimization using constant criterion surface algorithm. Bull Pol Acad Sci-Tech Sci 60(2):229-236

Osyczka A, Krenich S, Krzystek J, Habel J (2004) Evolutionary optimization system (EOS) for design automation. In: Burczynski T, Osyczka A (eds) Evolutionary methods in mechanics. Kluwer, Dordrecht

Querin OM, Steven GP, Xie YM (1998) Evolutionary structural optimization (ESO) using bidirectional algorithm. Eng Comput 15:1031-104

Rozvany GIN (1998) Exact analytical solutions for some popular benchmark problems in topology optimization. Struct Optim $15: 42-48$

Thomas H, Zhou M, Schramm U (2002) Issues of commercial optimization software development. Struct Optim 23:97-110

Tovar A, Patel NM, Niebur GL, Sen M, Renaud JE (2006) Topology optimization using a hybrid cellular automation method with local control rules. ASME J Mech Des 128:1205-1216

Wasiutynski Z (1960) On the congruency of the forming according to the minimum potential energy with that according to equal strength. Bull De L'Acad Pol des Sci. Ser Des Sci Tech 8(6):259268

Xie YM, Steven GP (1993) A simple evolutionary procedure for structural optimisation. Comput Struct 49:885-96

Xue X, Schmid F, Smith RA (2007) Analysis of the structural characteristics of an intermediate rail vehicle and their effect on vehicle crash performance. Proc IMechE Part F J Rail Rapid Transit 221:339-352

Zhou M, Rozvany GIN (1991) The COC algorithm, part II: topological, geometry and generalized shape optimization. Comput Methods Appl Mech Eng 89:197-224 